\title{
A novel fatty acid lipophilic index and risk of CHD in US men: the Health Professionals Follow-Up Study
}

\author{
Hongyu Wu ${ }^{1}$, Eric L. Ding ${ }^{1,2 *}$, Estefanía T. Toledo ${ }^{1,3}$, Hannia Campos ${ }^{1}$, Ana Baylin ${ }^{4}$, Frank B. Hu ${ }^{1,2,5}$ \\ and Qi Sun ${ }^{1,2 *}$ \\ ${ }^{1}$ Department of Nutrition, Harvard School of Public Health, 665 Huntington Avenue, Boston MA O2115, USA \\ ${ }^{2}$ Channing Division of Network Medicine, Department of Medicine, Brigham and Women's Hospital and Harvard Medical \\ School, Boston, MA, USA \\ ${ }^{3}$ Department of Preventive Medicine and Public Health, University of Navarra, Pamplona, Spain \\ ${ }^{4}$ Department of Epidemiology, School of Public Health, University of Michigan, Ann Arbor, MI, USA \\ ${ }^{5}$ Department of Epidemiology, Harvard School of Public Health, Boston, MA, USA
}

(Submitted 15 May 2012 - Final revision received 29 October 2012 - Accepted 29 October 2012 - First published online 8 January 2013)

\begin{abstract}
Few epidemiological studies have examined the association between an overall fatty acid (FA) profile and CHD risk. The aim of the present study was to examine a novel index that summarises individual FA levels based on FA affinity and fluidity in relation to CHD risk in men. In a prospective nested case-control study, FA in plasma and erythrocytes were measured in 459 CHD cases and 879 matched controls. Lipophilic index (LI) was computed by summing the products between FA levels and melting point of each FA to reflect the overall FA lipophilicity. Among controls, higher plasma LI was significantly correlated with adverse profiles of blood lipids, inflammatory markers and adiponectin. After multivariate adjustment for age, smoking, BMI and other CHD risk factors, plasma LI was significantly associated with an increased risk of CHD: the relative risk was 1.61 (95\% CI 1.03, 2.53; $P$ for trend=0.04) comparing extreme quintiles. This association was attenuated to $1.21(95 \%$ CI $0.48,3.09 ; P$ for trend=0.77) after adjusting for plasma levels of total trans-FA, long-chain $n$-3 FA and polyunsaturated:saturated fat ratio. Erythrocyte LI was not significantly associated with CHD risk. The present data indicate that a novel LI is associated with an adverse profile of cardiovascular risk markers and increased risk of CHD in men; its usefulness as a complement of individual FA in assessing disease risk needs to be elucidated in future studies.
\end{abstract}

Key words: Fatty acids: Lipophilic index: Lipophilicity: CHD

Fatty acids are a group of molecules sharing a common structure of a hydrophilic carboxyl terminal and a hydrophobic hydrocarbon chain that jointly endow fatty acids with their amphipathic nature. Because of this nature, fatty acids are essential components of phospholipids, which are building blocks of cellular or blood lipid membranes. Fatty acid composition in membranes determines the fluidity of the membranes, which subsequently influences the functions of membrane-bound proteins and cells ${ }^{(1)}$. Specifically, membrane fluidity is largely dictated by the lipophilic attraction among fatty acids in phospholipid layers, which depends primarily on the length of hydrocarbon chain and number of double bond of fatty acids. Fatty acids from both diet and de novo synthesis are incorporated into the membrane phospholipids and subsequently modify the membrane's viscosity as well as its functions ${ }^{(2)}$.
Multiple lines of evidence have suggested that the membrane fluidity of cells and lipoproteins may play a role in the aetiology of CHD through various pathways, such as effects on blood pressure, blood lipid metabolism and endothelial function ${ }^{(3-5)}$. Epidemiological data regarding membrane fluidity and CHD risk, however, are lacking because the present broad fatty acid classifications do not take into account their diverse biological properties such as affinity and fluidity. A unified approach to summarising an overall fatty acid profile that reflects membrane fluidity is needed to examine the hypothesis of interest.

We, therefore, developed a novel index ${ }^{(6)}$ to assess fatty acid lipophilicity by summarising levels of individual fatty acids and their melting points, which measure the lipophilic attraction between fatty acids ${ }^{(7)}$. In the present analysis, we aimed to prospectively evaluate this novel lipophilic index (LI)

Abbreviations: CRP, C-reactive protein; LI, lipophilic index; MI, myocardial infarction; RR, relative risk; TFA, trans-fatty acid. 
of fatty acids in plasma and erythrocyte membranes in relation to CHD risk among US men in a prospective nested casecontrol study within the Health Professionals Follow-up Study cohort.

\section{Research design and methods}

\section{Study population}

The Health Professionals Follow-up Study is an ongoing prospective cohort study consisting of 51529 US male health professionals who were aged 40-75 years at study inception in 1986. Medical history, lifestyle practices and diet were assessed at baseline and updated every 2-4 years using selfadministered questionnaires. In 1993-1995, a total of 18159 participants provided blood samples, which were centrifuged and aliquoted into cryotubes as plasma, buffy coat and erythrocytes upon arrival. All cryotubes were stored in the vapor phase of liquid $\mathrm{N}_{2}$ freezers at a temperature $\leq-130^{\circ} \mathrm{C}$. A nested case-control study of CHD was conducted among the participants who provided blood samples ${ }^{(8)}$. Briefly, among those who were free of CVD at blood draw, we prospectively identified incident CHD cases and selected one to two controls for each case using the risk-set sampling method from those who remained free of CHD events when the case was diagnosed. Cases and controls were matched on age ( \pm 2 years), smoking status (never smoke, past smoker, current smoker: 1-14 cigarettes/d, 15+ cigarettes/ d) and month of blood draw. Through 2008, a total of 460 CHD cases, including 358 cases of non-fatal myocardial infarction (MI) and 102 cases of fatal CHD, were identified and confirmed, and 894 controls were selected. All analyses were conducted for plasma and erythrocyte LI separately. A total of fourteen cases and fifty-six controls had missing plasma fatty acid levels and were excluded in plasma LI analyses, while two cases and eighteen controls had missing erythrocyte fatty acids and were excluded from erythrocyte LI analyses. After these exclusions, 446 CHD cases and 838 controls were included for plasma LI analyses, and $458 \mathrm{CHD}$ cases and 876 controls were included for erythrocyte LI analyses. Among these participants, a total of 787 men provided a fasting blood sample (time since last meal before blood draw $\geq 8 \mathrm{~h}$ ). There were no significant differences of plasma LI and erythrocyte LI values between fasting samples and non-fasting samples (18.46 v. 18.24 for plasma LI and 25.64 v. 25.67 for erythrocyte LI).

The study protocol was approved by the institutional review board of the Brigham and Women's Hospital and the Human Subjects Committee Review Board of Harvard School of Public Health.

\section{Assessment of CHD endpoint}

Study physicians who were blinded to exposure status reviewed medical records of participants who reported having MI in follow-up questionnaires. Non-fatal MI cases were confirmed using the WHO criteria, which require typical symptoms plus either electrocardiographic abnormality or elevated cardiac enzyme levels ${ }^{(9)}$. Fatal CHD was identified through reports from next of kin, from postal authorities or by searching the National Death Index. Fatal CHD was defined according to Internation Classification of Diseases (ICD)-9 codes 410-412 and was confirmed by reviewing hospital records or autopsy reports, if CHD was listed as the cause of death on the death certificate and if evidence of previous CHD was available in the medical records. For cases with $\mathrm{CHD}$ as the underlying cause on the death certificate, but no medical records concerning the death were available and no prior knowledge of CHD was indicated, we designated such cases as probable fatal CHD cases $^{(8)}$. We excluded disconfirmed CHD deaths in the case-control study. Because the exclusion of probable fatal CHD events $(n$ 9) did not alter the results, we included both confirmed and probable fatal CHD in the present analysis to maximise statistical power. Total CHD was defined as non-fatal MI plus fatal CHD.

\section{Fatty acid measurement and lipophilic index calculation}

Fatty acids of total plasma and erythrocyte membranes were measured by GLC, which has been described in detail elsewhere $^{(10)}$. Briefly, fatty acids were extracted from plasma and erythrocyte membranes using a hexane-isopropanol mixture and esterified with methanol and acetyl chloride. After esterification, the methanol and acetyl chloride were evaporated and the fatty acid methyl esters were redissolved in isooctane. The methyl esters were analysed using GLC. Peak retention times and area percentages of total fatty acids were identified by injecting known standards (Nu-Chek-Prep). A total of thirtyfive plasma fatty acids and thirty-six erythrocyte membrane fatty acids were identified. The content of each fatty acid was expressed as a percentage of total fatty acids.

Samples of matched case-control sets were handled identically and assayed in the same analytical run. Both technicians and laboratory personnel were blinded to case-control status of the samples. Laboratory control samples were run along with case-control samples. CV of the assay were assessed by repeatedly analysing quality-control samples. The average intra-assay CV was $13 \%$ for both plasma and erythrocyte fatty acids, with relative content higher than $0 \cdot 2 \%$. For those fatty acids with relative content lower than $0 \cdot 2 \%$, the average CV was 22 and 30\% for plasma and erythrocyte fatty acids, respectively. The intra-assay $\mathrm{CV}$ for individual fatty acids are shown in Supplementary Table S1 (available online).

The LI for plasma and erythrocyte fatty acid composition were calculated as a summation of the product of the levels of fatty acids (percentage of total fatty acid) and the melting point ${ }^{(11)}$ of each fatty acid (in ${ }^{\circ} \mathrm{C}$ ) using the following equation:

lipophilic index $=\frac{\left.\sum_{K} \text { [Levels of fatty acid }(\%) i \times \text { melting point }\left({ }^{\circ} \mathrm{C}\right) i\right]}{\sum_{K}[\text { Levels of fatty } \operatorname{acid}(\%) i]}$,

where $i$ is the individual fatty acid and $k$ is the number of fatty acids used to calculate LI. This index reflects the overall fatty acid lipophilicity in plasma or erythrocytes. 
In the present study, all fatty acids with available melting points ( $n$ 26), including 14:0,15:0,16:0,17:0,18:0,19:0, $20: 0, \quad 22: 0, \quad 23: 0, \quad 24: 0, \quad 16: 1 n-7, \quad 18: 1 n-7, \quad 18: 1 n-9$, $20: 1 n-9, \quad 24: 1 n-9, \quad 18: 2 n-6, \quad 18: 3 n-6, \quad 20: 4 n-6, \quad 18: 3 n-3$ ( $\alpha$-linolenicacid), $20: 5 n$-3(EPA), 22:6n-3(DHA), trans-16:1n7, total trans-18:1 isomers and three trans-18:2 isomers (9t,12t-18:2n-6, 9c,12t-18:2n-6 and 9t,12c-18:2n-6) were used to compute fatty acid LI in plasma and erythrocytes. Because individual trans-18:1 isomers were not separated, we used the mean melting point of trans-18:1n-12, trans$18: 1 n-9$ and trans-18:1n-7 as the melting point of total trans-18:1. The melting points for selected fatty acids range from $52 \cdot 3$ to $87 \cdot 8^{\circ} \mathrm{C}$ for SFA, 0 to $42 \cdot 8^{\circ} \mathrm{C}$ for MUFA, $-54 \cdot 1$ to $-5.0^{\circ} \mathrm{C}$ for PUFA and 1.0 to $48.7^{\circ} \mathrm{C}$ for trans-fatty acids (TFA). These fatty acids covered the majority of total fatty acids in plasma (96\%) and erythrocyte membranes (92\%).

\section{Measurement of plasma lipids and inflammatory markers}

Concentrations of total cholesterol, TAG and HDL-cholesterol were measured on the Hitachi 911 analyser using reagents and calibrators from Roche Diagnostics; all CV were $<1.8 \%$. LDL-cholesterol levels were assessed using a homogeneous direct method from Genzyme; CV was $<3 \cdot 1 \%$. C-reactive protein (CRP) was measured by the latex-enhanced immunoturbidimetric assay from Denka Seiken on the Hitachi 911 system and CV was $<2 \cdot 8 \%$. Soluble TNF- $\alpha$ receptors 1 and 2 and IL-6 levels were measured using ELISA from R\&D Systems with $\mathrm{CV}$ of $3 \cdot 5-9 \cdot 0 \%{ }^{(12)}$. Intercellular adhesion molecule-1 and vascular cell adhesion molecule- 1 were measured using a commercial ELISA (R\&D Systems); CV were $<3.6 \%$. Plasma adiponectin concentrations were measured by competitive radioimmunoassay (Linco Research) with a CV of $3 \cdot 4 \%{ }^{(13)}$.

\section{Assessment of covariates}

Information about weight, smoking status, aspirin use, cholesterol-lowering medication use and physical activity was inquired about in the baseline questionnaire. BMI was calculated as weight $/$ height $^{2}\left(\mathrm{~kg} / \mathrm{m}^{2}\right)$. Physical activity was expressed as metabolic equivalents per week using the duration of moderate or vigorous forms of exercise multiplied by the intensity of the activity ${ }^{(14)}$. History of hypertension, hypercholesterolaemia and diabetes and family history of MI were based on self-reports. Total energy intake and consumptions of carbohydrate, fats and alcohol were estimated using a validated FFQ. Alternate Healthy Eating Index score was calculated based on intakes of fruit, vegetable, nuts, trans-fat, the ratio of PUFA:SFA and cereal fibre ${ }^{(15)}$.

\section{Statistical analysis}

Among controls, linear regression analysis was applied to examine the associations between individual fatty acids and LI. We calculated partial Spearman correlation coefficients $(r)$, which were adjusted for matching factors to evaluate the correlations between LI and blood lipids, inflammatory markers and other cardiovascular risk factors. We used conditional logistic regression to examine the association between LI and risk of CHD. Participants were categorised into quintiles based on the distribution of LI among the controls. Besides matching factors, we further controlled for physical activity (in quartiles), alcohol intake (none, 0.1-4.9, 5.0-9.9, 10.0-14.9, $15 \cdot 0+\mathrm{g} / \mathrm{d}$ ), BMI (continuous), family history of MI (yes/ no), history of hypertension (yes/no), history of hypercholesterolaemia (yes/no), aspirin use (yes/no), cholesterol-lowering medication use (yes/no), hypertension medication use (yes/no) and Alternate Healthy Eating Index score. Tests for linear trends were conducted by treating the median value for each quintile of LI as a continuous variable.

All $P$ values were two-sided, and 95\% CI were calculated for relative risks (RR). Data were analysed with the Statistical Analysis Systems software package, version 9.1 (SAS Institute, Inc.).

\section{Results}

The associations between LI and individual fatty acids among controls are shown in Table 1 . Both plasma and erythrocyte LI were positively associated with individual SFA, including $14: 0,15: 0,16: 0,17: 0,18: 0,19: 0,20: 0,22: 0,23: 0$ and $24: 0$ (all $\beta \geq 0 \cdot 17, P<0 \cdot 001$ ). In contrast, LI were inversely correlated with all PUFA, including $\alpha$-linolenic acid, EPA, DHA, $18: 2 n-6, \quad 18: 3 n-6$ and $20: 4 n-6$ (all $\beta \leq-0 \cdot 25$, $P<0.001)$. Both plasma and erythrocyte LI showed inverse association with MUFA, including 16:1n-7, 18:1n-7 and 18:1n-9 (all $\beta \leq-0.03, \quad P<0.001$ ), except $24: 1 n-9$ ( $\beta=0.26$ for plasma LI and 0.18 for erythrocyte LI, $P<0 \cdot 001)$. The correlations with plasma and erythrocyte LI were not consistent among TFA. For example, both plasma and erythrocyte LI were positively correlated with total trans-18: 1 isomers $(\beta=0.31$ for plasma LI and 0.26 for erythrocyte LI, $P<0.001)$, whereas inverse correlations were observed for $9 c, 12 t-18: 2 n-6$ and $9 t, 12 c-18: 2 n-6$ isomers ( $\beta=-0.20$ and $-0 \cdot 18$ for plasma LI and -0.35 and $-0 \cdot 16$ for erythrocyte LI, $P<0 \cdot 001$ ). Plasma LI, but not erythrocyte LI, was also significantly correlated with levels of plasma trans-16:1n-7 ( $\beta=0 \cdot 10$ for plasma LI, $P<0 \cdot 001)$. Using the forward selection procedure of linear regression, we found that the strongest determinants of plasma LI were $16: 0$, total trans-18:1 isomers, 18:0, 20:4n-6, 18:1n-9 and DHA in plasma, whereas the major determinants of erythrocyte LI were $18: 0,14: 0$, DHA, $18: 2 n-6,20: 4 n-6$ and $18: 1 n-9$ in erythrocyte membranes. The correlation coefficient between plasma LI and erythrocyte LI was $0 \cdot 13(P<0 \cdot 001)$.

Associations between baseline lifestyle and dietary factors and plasma or erythrocyte LI among control participants are shown in Table 2. Both plasma and erythrocyte LI were consistently correlated with less favourable risk factors of CHD, including higher BMI and lower levels of physical activity and Alternate Healthy Eating Index score. In addition, participants with higher plasma LI levels were more likely to be current smokers and have a history of hypertension and hypercholesterolaemia. Erythrocyte LI levels were not correlated with these factors. Both plasma and erythrocyte LI were weakly associated with higher intakes of SFA $(r 0 \cdot 16$, 
Table 1. Multiple linear regression analysis of selected fatty acids in relation to lipophilic index: the Health Professionals Follow-up Study*

(Mean values, standard deviations, $\beta$ values and standard errors)

\begin{tabular}{|c|c|c|c|c|c|c|c|c|c|}
\hline \multirow[b]{2}{*}{ Variable } & \multirow[b]{2}{*}{ Melting point $\left({ }^{\circ} \mathrm{C}\right)$} & \multicolumn{4}{|c|}{ Plasma lipophilic index ( $n$ 838) } & \multicolumn{4}{|c|}{ Erythrocyte lipophilic index ( $n$ 876) } \\
\hline & & Mean & SD & $\beta$ & SE & Mean & SD & $\beta$ & SE \\
\hline \multicolumn{10}{|l|}{ SFA } \\
\hline $14: 0$ & 53.9 & 0.53 & 0.38 & 0.37 & 0.004 & 0.30 & 0.22 & 0.17 & 0.018 \\
\hline $15: 0$ & $52 \cdot 3$ & 0.14 & 0.05 & 0.30 & 0.025 & 0.11 & 0.06 & 0.50 & 0.043 \\
\hline $16: 0$ & $63 \cdot 1$ & $19 \cdot 1$ & $2 \cdot 46$ & 0.46 & 0.002 & $19 \cdot 2$ & 1.97 & 0.41 & 0.002 \\
\hline $17: 0$ & $61 \cdot 3$ & 0.31 & 0.09 & 0.44 & 0.012 & 0.37 & $0 \cdot 10$ & 0.39 & 0.041 \\
\hline $18: 0$ & $69 \cdot 6$ & $8 \cdot 17$ & 1.33 & 0.52 & 0.002 & 14.5 & $2 \cdot 26$ & 0.46 & 0.003 \\
\hline $19: 0$ & $68 \cdot 6$ & 0.10 & 0.05 & 0.50 & 0.022 & 0.12 & 0.04 & 0.37 & 0.035 \\
\hline $20: 0$ & $76 \cdot 8$ & 0.19 & 0.05 & 0.59 & 0.025 & 0.38 & 0.05 & 0.69 & 0.039 \\
\hline $22: 0$ & 81.5 & 0.49 & $0 \cdot 19$ & 0.66 & 0.009 & 1.58 & 0.28 & 0.63 & 0.011 \\
\hline $23: 0$ & $79 \cdot 1$ & 0.20 & 0.08 & 0.61 & 0.024 & 0.28 & 0.06 & 0.31 & 0.05 \\
\hline $24: 0$ & $87 \cdot 8$ & 0.42 & $0 \cdot 16$ & 0.71 & 0.012 & 3.90 & 0.81 & 0.68 & 0.005 \\
\hline \multicolumn{10}{|l|}{ MUFA } \\
\hline $16: 1 n-7$ & 0.0 & 1.55 & 0.79 & -0.20 & 0.003 & 0.46 & 0.23 & -0.24 & 0.012 \\
\hline $18: 1 n-7$ & $15 \cdot 0$ & 1.65 & 0.29 & -0.04 & 0.005 & 1.08 & 0.14 & -0.18 & 0.016 \\
\hline $18: 1 n-9$ & $16 \cdot 0$ & $19 \cdot 4$ & 3.01 & -0.03 & 0.002 & $13 \cdot 1$ & $2 \cdot 07$ & -0.1 & 0.002 \\
\hline $20: 1 n-9$ & $23 \cdot 3$ & 0.15 & 0.07 & $-0.01 \dagger$ & 0.017 & $0 \cdot 19$ & 0.04 & $-0.05 \dagger$ & 0.047 \\
\hline $24: 1 n-9$ & $42 \cdot 8$ & 0.54 & 0.23 & 0.26 & 0.007 & $3 \cdot 77$ & 0.80 & 0.18 & 0.004 \\
\hline \multicolumn{10}{|l|}{ PUFA } \\
\hline $18: 2 n-6$ & -5.0 & 30.6 & 4.45 & -0.25 & 0.002 & $13 \cdot 2$ & 3.07 & -0.33 & 0.002 \\
\hline $18: 3 n-6$ & $-11 \cdot 2$ & 0.42 & 0.15 & -0.31 & 0.008 & 0.1 & 0.04 & -0.54 & 0.042 \\
\hline $20: 4 n-6$ & -49.5 & $7 \cdot 29$ & 1.78 & -0.71 & 0.002 & 13.0 & 1.82 & -0.81 & 0.003 \\
\hline $18: 3 n-3$ & $-11 \cdot 2$ & 0.60 & 0.24 & -0.31 & 0.004 & 0.21 & 0.24 & -0.37 & 0.008 \\
\hline $20: 5 n-3$ & $-54 \cdot 1$ & 0.65 & 0.48 & -0.75 & 0.003 & 0.52 & 0.31 & -0.84 & 0.007 \\
\hline $22: 6 n-3$ & $-44 \cdot 2$ & $1 \cdot 75$ & 0.77 & -0.65 & 0.002 & $3 \cdot 7$ & $1 \cdot 22$ & -0.76 & 0.002 \\
\hline \multicolumn{10}{|l|}{ Trans-fatty acids } \\
\hline trans-16:1n-7 & 31.0 & 0.15 & 0.06 & 0.11 & 0.018 & 0.14 & 0.05 & $0.02 \dagger$ & 0.05 \\
\hline trans-18: 1 & 48.7 & 1.74 & 1.01 & 0.31 & 0.002 & 1.48 & 0.64 & 0.26 & 0.004 \\
\hline $9 t, 12 t-18: 2 n-6$ & 28.5 & 0.04 & 0.03 & 0.22 & 0.027 & 0.01 & 0.02 & $-0.02 \dagger$ & 0.075 \\
\hline $9 c, 12 t-18: 2 n-6$ & 1.0 & 0.24 & 0.11 & -0.20 & 0.01 & $0 \cdot 11$ & 0.04 & -0.35 & 0.083 \\
\hline $9 t, 12 c-18: 2 n-6$ & 1.0 & $0 \cdot 16$ & $0 \cdot 11$ & -0.18 & 0.01 & 0.08 & 0.04 & -0.16 & 0.084 \\
\hline
\end{tabular}

${ }^{*}$ All $P<0.001$ unless otherwise indicated.

†Values were non-significant.

$P<0.001$ for both LI), lower intakes of PUFA $(r-0.14$ for plasma LI and $r-0.16$ for erythrocyte LI, $P<0.001)$ and lower dietary PUFA:SFA ratio $(r-0.15$ for plasma LI and $r-0.17$ for erythrocyte LI, $P<0.001)$. We did not observe significant association between LI and intakes of MUFA or TFA. We observed moderate correlations between dietary LI and LI of plasma and erythrocyte: the correlation coefficient was $0 \cdot 16$ for plasma LI and $0 \cdot 17$ for erythrocyte LI $(P<0 \cdot 001$ for both correlations).

Supplementary Table 2 (available online) presents partial Spearman correlations of plasma and erythrocyte LI with blood lipids and other CHD risk markers among controls. After adjusting for matching factors, including age, smoking and month of blood draw, higher plasma LI was significantly correlated with adverse profiles of CHD risk factors, including elevated levels of TAG $(r \quad 0.58, \quad P<0.001)$, total:HDLcholesterol ratio $(r 0.33, P<0.001)$, LDL:HDL-cholesterol ratio $(r 0 \cdot 14, P<0 \cdot 001)$, CRP $(r 0 \cdot 10, P=0 \cdot 003)$, IL-6 $(r 0 \cdot 18$, $P<0 \cdot 001), \quad$ TNF- $\alpha$ receptor $1 \quad(r \quad 0 \cdot 18, \quad P<0 \cdot 001), \quad$ TNF- $\alpha$ receptor $2(r 0.13, P=0.005)$, intercellular adhesion molecule-1 $(r 0 \cdot 10, P=0 \cdot 02)$, vascular cell adhesion molecule-1 ( $r$ 0.12, $P=0 \cdot 008)$, lower levels of HDL-cholesterol $(r-0 \cdot 37$, $P<0.001)$ and adiponectin $(r-0.21, \quad P<0.001)$. These correlations were much weaker for erythrocyte LI. Both plasma and erythrocyte LI were inversely correlated with LDL-cholesterol ( $r-0 \cdot 18$ and $-0 \cdot 11$, respectively, $P<0 \cdot 002)$.

The baseline characteristics of cases and controls are presented in Supplementary Table 3 (available online). The associations of plasma and erythrocyte LI with risk of CHD are shown in Table 3. In a crude model that controlled for matching factors only, the RR was 1.76 (95\% CI 1.15, 2.69; $P$ for trend=0.01), comparing the highest with the lowest quintile of plasma LI. After multivariable adjustment of CHD risk factors, including $\mathrm{BMI}$ and lifestyle practices, the RR comparing extreme quintiles was attenuated to $1.61(95 \% \mathrm{CI}$ 1.03, 2.53; $P$ for trend=0.04). In a sub-group analysis, we found that the association for plasma LI was more pronounced for non-fatal MI (RR 1.81, 95\% CI 1.09, 3.01; $P$ for trend=0.01) in comparison with that for fatal CHD (RR $1 \cdot 05,95 \%$ CI 0.33 , 3.33; $P$ for trend=0.67). Although a positive trend was also observed for erythrocyte LI, the association did not achieve statistical significance.

In a secondary analysis, we explored whether the association between plasma LI and CHD was independent of plasma levels of fatty acids that are established risk factors of CHD, such as PUFA:SFA ratio, long-chain $n-3$ fatty acids (EPA and DHA) and total TFA. The multivariable-adjusted RR comparing extreme quintile of plasma levels of PUFA:SFA 
Table 2. Baseline characteristics of control participants by quintiles (Q) of lipophilic index at baseline in 1994, the Health Professionals Follow-up Study (Mean values, standard deviations and percentages)

\begin{tabular}{|c|c|c|c|c|c|c|c|c|c|c|c|c|}
\hline \multirow[b]{3}{*}{ Characteristics } & \multicolumn{6}{|c|}{ Plasma lipophilic index } & \multicolumn{6}{|c|}{ Erythrocyte lipophilic index } \\
\hline & \multicolumn{2}{|c|}{ Q1 } & \multicolumn{2}{|c|}{ Q3 } & \multicolumn{2}{|c|}{ Q5 } & \multicolumn{2}{|c|}{ Q1 } & \multicolumn{2}{|c|}{ Q3 } & \multicolumn{2}{|c|}{ Q5 } \\
\hline & Mean & SD & Mean & SD & Mean & SD & Mean & SD & Mean & SD & Mean & SD \\
\hline No. of participants & $167 \cdot 0$ & & $168 \cdot 0$ & & $167 \cdot 0$ & & $175 \cdot 0$ & & $176 \cdot 0$ & & $175 \cdot 0$ & \\
\hline Plasma lipophilic index & 14.6 & 1.0 & 17.9 & 0.4 & $22.5^{*}$ & $2 \cdot 2$ & $17 \cdot 4$ & $2 \cdot 7$ & 18.5 & $2 \cdot 7$ & $18 \cdot 5^{\star}$ & $3 \cdot 0$ \\
\hline Erythrocyte lipophilic index & $25 \cdot 5$ & 3.3 & $25 \cdot 7$ & $2 \cdot 9$ & $25 \cdot 8$ & $2 \cdot 0$ & $22 \cdot 8$ & 1.2 & $25 \cdot 2$ & 0.3 & $29 \cdot 7^{*}$ & 3.3 \\
\hline \multicolumn{13}{|l|}{ Demographic characteristics } \\
\hline Age (years) $\dagger$ & 63.3 & 8.7 & $63 \cdot 3$ & $8 \cdot 8$ & 65.0 & 7.9 & 63.6 & 8.9 & 63.8 & 8.5 & 63.2 & $8 \cdot 8$ \\
\hline BMI $\left(\mathrm{kg} / \mathrm{m}^{2}\right)$ & 24.7 & $2 \cdot 3$ & $25 \cdot 4$ & $2 \cdot 8$ & $26 \cdot 2^{*}$ & $3 \cdot 2$ & $25 \cdot 0$ & $2 \cdot 9$ & $25 \cdot 8$ & $2 \cdot 7$ & $25 \cdot 7^{*}$ & 3.4 \\
\hline Physical activity (MET-h/week) & 23.2 & $26 \cdot 9$ & $19 \cdot 1$ & 22.5 & $15 \cdot 9$ & $17 \cdot 4$ & $22 \cdot 8$ & 43.2 & 21.5 & $19 \cdot 4$ & $17 \cdot 6$ & 20.9 \\
\hline Current smoker $(\%) \dagger$ & \multicolumn{2}{|c|}{$\begin{array}{c}4.3 \\
22.8\end{array}$} & \multicolumn{2}{|c|}{8.9} & \multicolumn{2}{|c|}{$15 \cdot 2^{*}$} & \multicolumn{2}{|c|}{6.0} & \multicolumn{2}{|c|}{$12 \cdot 4$} & \multicolumn{2}{|c|}{9.9} \\
\hline History of hypertension (\%) & \multirow{2}{*}{\multicolumn{2}{|c|}{$\begin{array}{l}22 \cdot 8 \\
38 \cdot 3\end{array}$}} & \multirow{2}{*}{\multicolumn{2}{|c|}{$\begin{array}{l}23.2 \\
38.7\end{array}$}} & \multirow{2}{*}{\multicolumn{2}{|c|}{$40 \cdot 7^{\star}$}} & & & \multicolumn{2}{|c|}{$25 \cdot 6$} & \multicolumn{2}{|c|}{31.4} \\
\hline History of high cholesterol (\%) & & & & & & & & & \multicolumn{2}{|c|}{37.5} & \multicolumn{2}{|c|}{$38.9 \ddagger$} \\
\hline Family history of $\mathrm{Ml}(\%)$ & \multicolumn{2}{|c|}{31.7} & \multicolumn{2}{|c|}{29.8} & \multicolumn{2}{|c|}{37.1} & & & & & & \\
\hline Hypertension medication user (\%) & \multirow{2}{*}{\multicolumn{2}{|c|}{$18 \cdot 6$}} & \multicolumn{2}{|c|}{$14 \cdot 3$} & & & & & & & & \\
\hline Cholesterol-lowering medication user (\%) & & & & & & & & & & & & \\
\hline Aspirin user (\%) & & & & & & & & & & & & \\
\hline Diet & & & & & & & & & & & & \\
\hline Total energy intake $(\mathrm{kJ} / \mathrm{d})$ & 8501.5 & $2446 \cdot 0$ & $8519 \cdot 0$ & $2684 \cdot 0$ & $8696 \cdot 0$ & $2924 \cdot 2$ & $8332 \cdot 0$ & $2332 \cdot 2$ & $8548 \cdot 3$ & $2568 \cdot 1$ & $8570 \cdot 9$ & $2912 \cdot 9$ \\
\hline Total carbohydrate intake $(\mathrm{g} / \mathrm{d})$ & $246 \cdot 2$ & 38.0 & $242 \cdot 2$ & $39 \cdot 6$ & $244 \cdot 7$ & 41.9 & $249 \cdot 1$ & 37.7 & $244 \cdot 0$ & 42.5 & $240 \cdot 1^{*}$ & $38 \cdot 8$ \\
\hline Glycaemic load & $130 \cdot 5$ & $22 \cdot 6$ & $128 \cdot 1$ & $23 \cdot 2$ & 129.5 & $25 \cdot 2$ & $131 \cdot 0$ & $21 \cdot 7$ & 128.7 & 24.9 & $126 \cdot 7^{*}$ & $22 \cdot 6$ \\
\hline Total fat intake $(\mathrm{g} / \mathrm{d})$ & 68.5 & $12 \cdot 0$ & $70 \cdot 0$ & $12 \cdot 7$ & $68 \cdot 2$ & $13 \cdot 2$ & $66 \cdot 6$ & $12 \cdot 3$ & 68.4 & $12 \cdot 6$ & $72 \cdot 1^{*}$ & $12 \cdot 5$ \\
\hline Saturated fat intake (\% total fat) & $32 \cdot 1$ & 3.6 & 33.6 & 3.6 & $34.0^{*}$ & 3.8 & 32.4 & 3.6 & 33.2 & 3.8 & $34 \cdot 2^{*}$ & 3.4 \\
\hline Monounsaturated fat intake ( $\%$ total fat) & $39 \cdot 1$ & 1.8 & $39 \cdot 1$ & $2 \cdot 2$ & $38 \cdot 8$ & $2 \cdot 1$ & 38.9 & $2 \cdot 1$ & 38.9 & $2 \cdot 0$ & 38.9 & $2 \cdot 0$ \\
\hline Polyunsaturated fat intake ( $\%$ total fat) & 19.7 & $3 \cdot 2$ & $18 \cdot 2$ & 3.1 & $18 \cdot 1^{*}$ & $3 \cdot 3$ & $19 \cdot 6$ & $3 \cdot 3$ & $18 \cdot 8$ & $3 \cdot 3$ & $17 \cdot 9^{*}$ & $2 \cdot 9$ \\
\hline Trans-fat intake (\% total fat) & 4.3 & $1 \cdot 1$ & 4.2 & $1 \cdot 2$ & 4.5 & 1.3 & $4 \cdot 2$ & 1.3 & $4 \cdot 2$ & $1 \cdot 2$ & 4.4 & $1 \cdot 1$ \\
\hline$P: S$ ratio & 0.54 & 0.20 & 0.46 & 0.17 & $0.46^{*}$ & 0.16 & 0.53 & $0 \cdot 19$ & 0.49 & 0.19 & $0.44^{*}$ & 0.14 \\
\hline Alcohol $(\mathrm{g} / \mathrm{d})$ & $11 \cdot 8$ & $13 \cdot 0$ & $12 \cdot 9$ & 13.8 & 13.5 & $16 \cdot 5$ & 13.6 & $15 \cdot 2$ & 14.5 & $16 \cdot 2$ & $10 \cdot 9$ & $14 \cdot 0$ \\
\hline AHEI diet score & 43.9 & 8.9 & 41.6 & 9.5 & $41.0^{*}$ & 9.4 & 43.9 & 9.8 & $42 \cdot 3$ & 9.5 & $40.0^{*}$ & 7.8 \\
\hline
\end{tabular}

MET, metabolic equivalents; MI, myocardial infarction; P:S, PUFA:SFA; AHEl, Alternate Healthy Eating Index.

${ }^{\star} P$ for trend $<0.01$.

† Age and smoking status are matching factors.

$\ddagger P$ for trend $<0.05$ 
ratio, long-chain $n-3$ fatty acids and total TFA were 0.61 (95\% CI $0 \cdot 40,0 \cdot 95, P$ for trend=0.04), 0.64 (95\% CI $0.42,1.00, P$ for trend $=0.04$ ) and 1.45 (95\% CI $0.94,2 \cdot 23, P$ for trend $=0 \cdot 14$ ), respectively. After adjusting for these three aforementioned factors, the RR comparing extreme quintiles of plasma LI was attenuated to 1.21 (95\% CI 0.48, 3.09; $P$ for trend=0.77). In a separate analysis, we evaluated whether the association of plasma LI was driven by fatty acids that were the strongest predictors of plasma LI identified in the forward selection analysis, i.e. $16: 0$, total trans-18: 1 isomers, $18: 0,20: 4 n-6,18: 1 n-9$ and DHA. The RR of total CHD comparing extreme quintiles were 1.42 (95\% CI $0.77,2.60), 1.63$ (95\% CI 1.02, 2.61), $1.67(95 \%$ CI $1.07,2.64), 1.30$ (95\% CI $0.75,2.27), 1.20$ (95\% CI 0.71 , $2.05)$ and 1.44 (95\% CI 0.89, 2.35) after further adjustment for $16: 0$, total trans-18:1 isomers, $18: 0,20: 4 n-6,18: 1 n-9$ and DHA, respectively. The association was substantially attenuated when all six plasma fatty acids were adjusted simultaneously; the RR was $1 \cdot 04$ (95\% CI 0.37, 2.94) comparing extreme quintiles. Lastly, we examined whether plasma lipids and inflammatory markers explained the associations of interest. Further adjustment for TAG and HDL-cholesterol attenuated the association between plasma LI and CHD: the RR of total CHD comparing extreme quintiles was 1.11 (95\% CI 0.67, 1.86;
$P$ for trend=0.77). Adjustment for CRP, IL-6, TNF- $\alpha$ receptor 1 , TNF- $\alpha$ receptor 2 , intercellular adhesion molecule-1 or vascular cell adhesion molecule-1 somewhat strengthened the association. The RR of total CHD comparing extreme quintiles were 1.59 (95\% CI 1.01, 2.49) with CRP adjustment, 1.90 (95\% CI 1.04, 3.48) with IL-6 adjustment, (1.90 95\% CI $1.04,3.47)$ with $\mathrm{TNF}-\alpha$ receptor 1 adjustment, 1.89 (95\% CI 1.03, 3.47) with TNF- $\alpha$ receptor 2 adjustment, 1.88 (95\% CI 1.02, 3.44) with intercellular adhesion molecule-1 adjustment and 1.84 (1.01, 3.37) with vascular cell adhesion molecule-1 adjustment.

\section{Discussion}

In the present nested case-control study among US men, we examined a novel fatty acid LI that represents overall lipophilicity of fatty acids in plasma or erythrocyte membranes in relation to CHD risk. Plasma LI was significantly associated with higher risk of developing CHD, especially non-fatal MI. Further adjustment for fatty acids that were established risk factors of CHD and blood lipids substantially attenuated the association between plasma LI and CHD. The associations were weaker for erythrocyte LI.

Table 3. CHD across the quintiles $(\mathrm{Q})$ of plasma and erythrocyte lipophilic index: the Health Professionals Follow-up Study*

(Relative risks (RR) and $95 \%$ confidence intervals)

\begin{tabular}{|c|c|c|c|c|c|c|c|c|c|c|}
\hline & Q1 & \multicolumn{2}{|r|}{ Q2 } & \multicolumn{2}{|r|}{ Q3 } & \multicolumn{2}{|r|}{ Q4 } & \multicolumn{2}{|r|}{ Q5 } & \multirow[b]{2}{*}{$P$ for trend } \\
\hline & $95 \% \mathrm{Cl}$ & $\mathrm{RR}$ & $95 \% \mathrm{Cl}$ & $\mathrm{RR}$ & $95 \% \mathrm{Cl}$ & $\mathrm{RR}$ & $95 \% \mathrm{Cl}$ & $\mathrm{RR}$ & $95 \% \mathrm{Cl}$ & \\
\hline \multicolumn{11}{|c|}{ Plasma lipophilic index } \\
\hline Median & $15 \cdot 0$ & & $16 \cdot 5$ & & $17 \cdot 9$ & & $19 \cdot 4$ & \multicolumn{2}{|c|}{$21 \cdot 7$} & \\
\hline Range & $10 \cdot 9-15 \cdot 8$ & \multicolumn{2}{|c|}{$15 \cdot 9-17 \cdot 2$} & \multicolumn{2}{|c|}{$17 \cdot 3-18 \cdot 6$} & \multicolumn{2}{|c|}{$18 \cdot 7-20 \cdot 3$} & \multicolumn{2}{|c|}{$20 \cdot 4-33 \cdot 4$} & \\
\hline \multicolumn{11}{|l|}{ Total CHD } \\
\hline Case/control & $74 / 167$ & \multicolumn{2}{|r|}{$81 / 168$} & \multicolumn{2}{|r|}{$83 / 168$} & \multicolumn{2}{|r|}{$95 / 168$} & \multicolumn{2}{|c|}{$113 / 167$} & \\
\hline Model 1† & 1.00 & $1 \cdot 27$ & $0.84,1.91$ & $1 \cdot 22$ & $0.81,1.85$ & 1.42 & $0.94,2.12$ & 1.76 & $1 \cdot 15,2 \cdot 69$ & 0.01 \\
\hline Model $2 \ddagger$ & $1 \cdot 00$ & $1 \cdot 23$ & $0.80,1.88$ & $1 \cdot 25$ & $0.81,1.93$ & $1 \cdot 34$ & $0.88,2.06$ & $1 \cdot 61$ & $1.03,2.53$ & 0.04 \\
\hline \multicolumn{11}{|l|}{ Non-fatal MI } \\
\hline Case/control & $60 / 130$ & \multicolumn{2}{|r|}{$63 / 133$} & \multicolumn{2}{|r|}{$65 / 124$} & \multicolumn{2}{|r|}{$75 / 124$} & \multicolumn{2}{|c|}{$81 / 120$} & \\
\hline Model 1† & 1.00 & $1 \cdot 16$ & $0.74,1.82$ & $1 \cdot 28$ & $0.81,2.02$ & 1.51 & $0.96,2.37$ & 1.78 & $1 \cdot 10,2 \cdot 86$ & 0.01 \\
\hline Model $2 \ddagger$ & 1.00 & $1 \cdot 19$ & $0.74,1.90$ & 1.40 & $0.86,2 \cdot 27$ & 1.52 & $0.94,2.45$ & 1.81 & $1.09,3.01$ & 0.01 \\
\hline \multicolumn{11}{|l|}{ Fatal CHD } \\
\hline Case/control & $14 / 26$ & \multicolumn{2}{|r|}{$18 / 21$} & \multicolumn{2}{|r|}{$18 / 31$} & \multicolumn{2}{|r|}{$20 / 38$} & \multicolumn{2}{|c|}{$32 / 34$} & \\
\hline Model 1† & 1.00 & 1.89 & $0.72,4.97$ & 1.05 & $0.39,2.82$ & $1 \cdot 14$ & $0.46,2.84$ & 1.68 & $0.65,4.35$ & 0.53 \\
\hline Model $2 \ddagger$ & 1.00 & 1.54 & $0.48,4.90$ & 0.85 & $0.27,2.65$ & 0.72 & $0.24,2 \cdot 13$ & 1.05 & $0.33,3.33$ & 0.67 \\
\hline \multicolumn{11}{|c|}{ Erythrocyte lipophilic index } \\
\hline Median & $23 \cdot 1$ & & $24 \cdot 3$ & & $25 \cdot 2$ & & $26 \cdot 2$ & & $28 \cdot 3$ & \\
\hline Range & $17 \cdot 0-23 \cdot 8$ & & $3 \cdot 9-24 \cdot 8$ & & $4.9-25 \cdot 6$ & & $5 \cdot 7-26 \cdot 9$ & & $0-42 \cdot 0$ & \\
\hline Total CHD & & & & & & & & & & \\
\hline Case/control & $78 / 175$ & & $97 / 175$ & & $95 / 176$ & & 100/175 & & $8 / 175$ & \\
\hline Model 1† & 1.00 & $1 \cdot 37$ & $0.91,2.08$ & 1.41 & $0.91,2.18$ & 1.58 & $1.01,2.49$ & 1.45 & $0.88,2.37$ & 0.22 \\
\hline Model 2‡ & 1.00 & 1.42 & $0.92,2.19$ & 1.58 & $1.00,2.50$ & 1.69 & $1.06,2 \cdot 70$ & 1.35 & $0.80,2.26$ & 0.41 \\
\hline Non-fatal MI & & & & & & & & & & \\
\hline Case/control & $65 / 142$ & & $74 / 134$ & & $71 / 129$ & & $72 / 133$ & & $4 / 124$ & \\
\hline Model 1† & 1.00 & $1 \cdot 22$ & $0.78,1.92$ & $1 \cdot 25$ & $0.77,2.01$ & 1.39 & $0 \cdot 86,2 \cdot 27$ & 1.47 & $0.87,2.49$ & 0.17 \\
\hline Model $2 \ddagger$ & 1.00 & $1 \cdot 28$ & $0.80,2.05$ & 1.43 & $0.87,2.35$ & 1.51 & $0.91,2.51$ & 1.44 & $0.83,2.51$ & 0.24 \\
\hline Fatal CHD & & & & & & & & & & \\
\hline Case/control & $13 / 28$ & & $23 / 27$ & & $24 / 39$ & & $28 / 30$ & & $4 / 32$ & \\
\hline Model 1† & 1.00 & $2 \cdot 65$ & $0.88,7.97$ & $2 \cdot 86$ & $0.87,9.39$ & $3 \cdot 39$ & $0.95,12.1$ & 1.39 & $0.32,6 \cdot 0$ & 0.92 \\
\hline Model 2‡ & 1.00 & 3.04 & $0.77,12.02$ & 3.75 & $0.85,16.64$ & 3.68 & $0.78,17.48$ & 1.02 & $0.16,6.40$ & 0.46 \\
\hline
\end{tabular}

MI, myocardial infarction.

* The RR values were estimated using conditional logistic regression. Missing values for plasma and erythrocyte lipophilic index were $n 54$ and $n 4$, respectively.

$\dagger$ Model 1: adjusted for matching factors included age (years), smoking status (never/current smoker/past smoker) and month of blood draw.

$\ddagger$ Model 2: multivariate RR values additionally adjusted for alcohol intake ( $/ \mathrm{d}$ : $0,0.1-4.9,5 \cdot 0-9.9,10.0-14.9$ and $>15$ ), physical activity (quartile), family history of Ml (yes/no), history of hypertension (yes/no), history of hypercholesterolaemia (yes/no), aspirin use (yes/no), cholesterol-lowering medication use (yes/no), antihypertensive medication use (yes/no). Alternate Healthy Eating Index and BMI. 
It is well known that membrane lipid contents determine membrane fluidity or miscibility and, subsequently, the membrane's physiological function ${ }^{(16)}$. The inter-fatty acids attraction, one of the main factors regulating membrane fluidity, is determined by the van der Waals interaction, which depends on two main molecular characteristics of fatty acids: the length of the fatty acid hydrocarbon chain and fatty acid unsaturation, i.e. the number of double bonds ${ }^{(16)}$. With the exception of TFA, which have an overall linear structure because of the trans configuration of double bonds, longer hydrocarbon chain and more saturation will lead to tighter molecular packing or more lipophilic attraction. Such lipophilic attraction is reliably measured by the melting point of fatty acids ${ }^{(7)}$. This is the basis of using melting points to derive our LI, which theoretically reflects the relative fluidity of cellular or lipoprotein membranes.

Membrane fluidity may influence CHD risk through multiple pathways. Changes of membrane fluidity would modulate the activity of proteins involved in ion transport, signal transduction, cell $\mathrm{Ca}^{2+}$ handling and intracellular $\mathrm{pH}$ regulation ${ }^{(17)}$, suggesting that decreased membrane fluidity may play an important role in the pathogenesis of hypertension ${ }^{(4,18,19)}$. In addition, reduced membrane fluidity could also significantly alter the vascular endothelial response to shear stress and impair endothelial cell wound closure ${ }^{(5)}$. Moreover, low membrane fluidity may interfere with Na-dependent D-glucose transport $^{(20,21)}$. There is also evidence indicating that membrane fluidity is strongly related to insulin sensitivity ${ }^{(20,22)}$. In fact, effects of Metformin in diabetes treatment may be partially via its effects on membrane fluidity ${ }^{(23)}$. In addition to cell membrane fluidity, the fluidity of lipoprotein membranes may also play a critical role in CHD aetiology. Fluidity of lipoproteins' phospholipid layer is known to be affected by phospholipid fatty acyl composition of lipoproteins ${ }^{(24)}$. Phospholipid fluidity of HDL particles regulates the activity of lecithin:cholesterol acyltransferase $e^{(25)}$ and the capacity of HDL to promote cholesterol efflux ${ }^{(26)}$.

Of note, in the present study, we only calculated lipophilic indices of total plasma and erythrocyte membranes, the most accessible tissues in large epidemiological studies involving hundreds to thousands of human participants. We found a positive association between plasma LI and CHD risk. Although plasma fatty acid content may not directly measure that of cell membrane involved in the aforementioned mechanisms, evidence suggests that the plasma phospholipid compartment actively exchanges phospholipids with plasma membranes of cells ${ }^{(27,28)}$. Therefore, plasma phospholipids may serve as a dynamic and readily available pool for cell membrane phospholipids and subsequently affect membrane fatty acid composition, resulting in a change of membrane fluidity. The null association observed for erythrocyte membrane LI was unexpected. Although apparently more data are needed to confirm these findings, several reasons may explain the null results. Erythrocytes are incapable of de novo phospholipid synthesis, and the major pathway for the renewal of erythrocyte phospholipids is direct exchange of phosphatidylcholine with surrounding plasma lipoproteins ${ }^{(27)}$. Therefore, in comparison to plasma, erythrocyte membrane phospholipids may serve as a secondary pool for exchanging phospholipids with other cells. In addition, erythrocyte fatty acid measurements had relatively high measurement error (CV was, on average, $27 \%$ higher than plasma fatty acids), which might be due to more difficulties in lipid extraction from erythrocytes than plasma. In prospective studies, such random measurement error will, in general, lead to attenuation of true association.

The possibility of alternative explanations that do not relate to membrane fluidity deserves discussion. In the present study, plasma and erythrocyte LI were significantly correlated with high SFA and low PUFA intakes. In addition, trans-18:1 isomers and DHA, fatty acids that are exclusively or largely from the diet, were among the strongest individual fatty acids that determine the levels of plasma LI. Intake of these fatty acids can affect CHD risk through multiple pathways. For example, dietary SFA significantly increased the risk of CHD primarily through effects on increasing plasma concentration of LDL-cholesterol ${ }^{(29,30)}$. In contrast, replacement of SFA with PUFA in the diet significantly reduced LDL-cholesterol concentration and CHD risk ${ }^{(30)}$. Dietary PUFA have also been indicated to alter fatty acids composition of tissue cell membrane phospholipids and lead to improved insulin sensitivity $^{(31-33)}$. In addition, long-chain $n$-3 fatty acid intakes were significantly associated with lower levels of TAG and inflammatory biomarkers, as well as improved blood pressure and vascular function ${ }^{(34)}$. Dietary consumption of trans-fat might also contribute to an increased risk of CHD via its adverse effects on blood lipids ${ }^{(35)}$. Lastly, it is possible that a highSFA and low-PUFA diet raises CHD risk through promoting thromboembolism. It has been observed that serum agglutination or creaming occurred after fat emulsions were administered intravenously to critically ill patients ${ }^{(36)}$, especially when CRP levels were high ${ }^{(36-38)}$, and such agglutination might lead to increased risk of developing thromboembolism ${ }^{(38)}$.

There are several limitations in the present analysis that should be discussed. First, plasma or erythrocyte LI may not be a direct measurement of membrane fluidity of various cell types and plasma lipoproteins. In the present study, the association of plasma LI was not independent of some individual fatty acids, such as trans-18: 1 isomers and DHA, or blood lipids. Therefore, plasma LI did not provide additional predictive value beyond blood lipids and individual fatty acids that were established risk factors of CHD. More data of direct measurements of cell/lipoprotein membrane LI are warranted to corroborate our observations. Second, we cannot exclude the possibility that the present findings are due to unmeasured and residual confounding that is intrinsic to any observational studies. Third, we only measured plasma and erythrocyte fatty acid levels at baseline. Because fatty acid contents may fluctuate through change of diet, it is likely that this snapshot of fatty acid composition measurement may not represent the longterm average values. In general, this type of measurement errors tends to nullify associations. Lastly, the generalisability of the present findings is restricted to white male health professionals.

In summary, we derived a novel LI that summarises the overall lipophilicity of major fatty acids in plasma and 
erythrocytes. In a prospective study of male US health professionals, we found a significant association between this index in plasma and an increased risk of CHD, and this association was primarily attributable to several individual fatty acids, including trans-18:1 isomers and DHA. More studies are warranted to corroborate the present findings and to elucidate whether the LI possesses values in predicting future CHD beyond those of some individual FA that are established risk factors of CHD.

\section{Supplementary material}

To view supplementary material for this article, please visit http://dx.doi.org/10.1017/S0007114512005272

\section{Acknowledgements}

The authors' responsibilities were as follows: H. W. analysed the data and drafted the manuscript. E. L. D. conceived and developed the concept of LI. F. B. H. and Q. S. designed the study and supervised data analysis. All authors contributed to the interpretation of results and the critical revision of the manuscript. The present study was supported by research grants HL60,712 and CA055,075 from the National Institutes of Health, a career development award K99HL098,459 from the National Heart, Lung, and Blood Institute (to Q. S.), career development awards from the American Heart Association and American Diabetes Association (to E. L. D.), a Rio Hortega post-residency fellowship of the Instituto de Salud Carlos III, Ministry of Economy and Competitiveness, Spanish Government (to E. T. T.). There is no relevant conflict of interest to disclose.

\section{References}

1. Cooper RA (1977) Abnormalities of cell-membrane fluidity in the pathogenesis of disease. $N$ Engl J Med 297, 371-377.

2. Hodson L, Skeaff CM \& Fielding BA (2008) Fatty acid composition of adipose tissue and blood in humans and its use as a biomarker of dietary intake. Prog Lipid Res 47, 348-380.

3. Jonas A, Zorich NL, Kezdy KE, et al. (1987) Reaction of discoidal complexes of apolipoprotein A-I and various phosphatidylcholines with lecithin cholesterol acyltransferase. Interfacial effects. J Biol Chem 262, 3969-3974.

4. Tsuda K \& Nishio I (2003) Membrane fluidity and hypertension. Am J Hypertens 16, 259-261.

5. Gojova A \& Barakat AI (2005) Vascular endothelial wound closure under shear stress: role of membrane fluidity and flow-sensitive ion channels. J Appl Physiol 98, 2355-2362.

6. Ding EL, Sun Q, Campos H, et al. (2008) Lipophilic index of fatty acid fluidity in erythrocyte and plasma and risk of coronary heart disease (abstract). Circulation 118, S_1089.

7. Vaclavik VA \& Christian EW (2008) Fats: fat and oil products. In Essentials of Food Science, pp. 271-310. New York, NY: Springer Science + Business Media, LLC.

8. Hatoum IJ, Cook NR, Nelson JJ, et al. (2011) Lipoproteinassociated phospholipase $\mathrm{A}_{2}$ activity improves risk discrimination of incident coronary heart disease among women. Am Heart J 161, 516-522.
9. Rose G \& Blackburn H (1982) Cardiovascular Survey Method, Monograph Series No. 56, 2nd ed. Geneva: World Health Organization.

10. Baylin A, Kabagambe EK, Siles X, et al. (2002) Adipose tissue biomarkers of fatty acid intake. Am J Clin Nutr 76, 750-757.

11. Japanese Conference on Biochemistry of Lipids. (2007) Lipid Bank for Web Database. http://lipidbank.jp/ (updated 2007). Japan Science and Technology Agency and International Medical Center of Japan.

12. Pischon T, Hu FB, Rexrode KM, et al. (2008) Inflammation, the metabolic syndrome, and risk of coronary heart disease in women and men. Atherosclerosis 197, 392-399.

13. Qi L, Rimm E, Liu S, et al. (2005) Dietary glycemic index, glycemic load, cereal fiber, and plasma adiponectin concentration in diabetic men. Diabetes Care 28, 1022-1028.

14. Wolf AM, Hunter DJ, Colditz GA, et al. (1994) Reproducibility and validity of a self-administered physical activity questionnaire. Int J Epidemiol 23, 991-999.

15. McCullough ML, Feskanich D, Stampfer MJ, et al. (2002) Diet quality and major chronic disease risk in men and women: moving toward improved dietary guidance. Am J Clin Nutr 76, 1261-1271.

16. van Meer G, Voelker DR \& Feigenson GW (2008) Membrane lipids: where they are and how they behave. Nat Rev Mol Cell Biol 9, 112-124.

17. Zicha J, Kunes J \& Devynck M-A (1999) Abnormalities of membrane function and lipid metabolism in hypertension - a review. Am J Hypertens 12, 315-331.

18. Tsuda K (2006) Adiponectin and membrane fluidity of erythrocytes in normotensive and hypertensive men. Obesity $\mathbf{1 4}$, $1505-1510$.

19. Tsuda K (2010) Oxidative stress and membrane fluidity of red blood cells in hypertensive and normotensive men: an electron spin resonance investigation. Int Heart $J \mathbf{5 1}$, 121-124.

20. Pilch PF, Thompson PA \& Czech MP (1980) Coordinate modulation of d-glucose transport activity and bilayer fluidity in plasma membranes derived from control and insulintreated adipocytes. Proc Natl Acad Sci U S A 77, 915-918.

21. Brasitus TA, Dudeja PK, Bolt MJ, et al. (1989) Dietary triacylglycerol modulates sodium-dependent d-glucose transport, fluidity and fatty acid composition of rat small intestinal brush-border membrane. Biochim Biophys Acta 979, $177-186$.

22. Muramatsu K, Maitani Y, Takayama K, et al. (1999) The relationship between the rigidity of the liposomal membrane and the absorption of insulin after nasal administration of liposomes modified with an enhancer containing insulin in rabbits. Drug Dev Ind Pharm 25, 1099-1105.

23. Wiernsperger NF \& Bailey CJ (1999) The antihyperglycaemic effect of metformin: therapeutic and cellular mechanisms. Drugs 58, Suppl. 1, 31-39, discussion 75-82.

24. Sola R, Baudet MF, Motta C, et al. (1990) Effects of dietary fats on the fluidity of human high-density lipoprotein: influence of the overall composition and phospholipid fatty acids. Biochim Biophys Acta 1043, 43-51.

25. Parks JS, Huggins KW, Gebre AK, et al. (2000) Phosphatidylcholine fluidity and structure affect lecithin:cholesterol acyltransferase activity. J Lipid Res 41, 546-553.

26. Berrougui H, Isabelle M, Cloutier M, et al. (2007) Age-related impairment of HDL-mediated cholesterol efflux. J Lipid Res 48, 328-336.

27. Reed CF (1968) Phospholipid exchange between plasma and erythrocytes in man and the dog. J Clin Invest 47, 749-760.

28. Emken EA, Rohwedder WK, Dutton HJ, et al. (1979) Incorporation of deuterium-labeled cis- and trans-9-octadecenoic 
acids in humans: plasma, erythrocyte, and platelet phospholipids. Lipids 14, 547-554.

29. Hu FB, Manson JE \& Willett WC (2001) Types of dietary fat and risk of coronary heart disease: a critical review. $J \mathrm{Am}$ Coll Nutr 20, 5-19.

30. Sacks FM \& Katan M (2002) Randomized clinical trials on the effects of dietary fat and carbohydrate on plasma lipoproteins and cardiovascular disease. Am J Med 113, Suppl. 9B, 13S-24S.

31. Field CJ, Ryan EA, Thomson AB, et al. (1990) Diet fat composition alters membrane phospholipid composition, insulin binding, and glucose metabolism in adipocytes from control and diabetic animals. $J$ Biol Chem 265, 11143-11150.

32. Ginsberg BH, Chatterjee P \& Yorek MA (1991) Insulin sensitivity is increased in Friend erythroleukemia cells enriched in polyunsaturated fatty acid. Receptor 1, 155-166.

33. Haugaard SB, Madsbad S, Hoy CE, et al. (2006) Dietary intervention increases $n-3$ long-chain polyunsaturated fatty acids in skeletal muscle membrane phospholipids of obese subjects. Implications for insulin sensitivity. Clin Endocrinol (Oxf) 64, 169-178.

34. Mozaffarian D \& Wu JH (5000) Omega-3 fatty acids and cardiovascular disease: effects on risk factors, molecular pathways, and clinical events. J Am Coll Cardiol 58, 2047-2067.

35. Mozaffarian D, Katan MB, Ascherio A, et al. (2006) Trans fatty acids and cardiovascular disease. $N$ Engl J Med 354, $1601-1613$.

36. Hulman G, Fraser I, Pearson HJ, et al. (1982) Agglutination of intralipid by sera of acutely ill patients. Lancet ii, $1426-1427$.

37. Lindh A, Johansson B, Lindholm M, et al. (1985) Agglutinate formation in serum samples mixed with intravenous fat emulsions. Crit Care Med 13, 151-154.

38. Hulman G (1995) The pathogenesis of fat embolism. J Pathol 176, 3-9. 